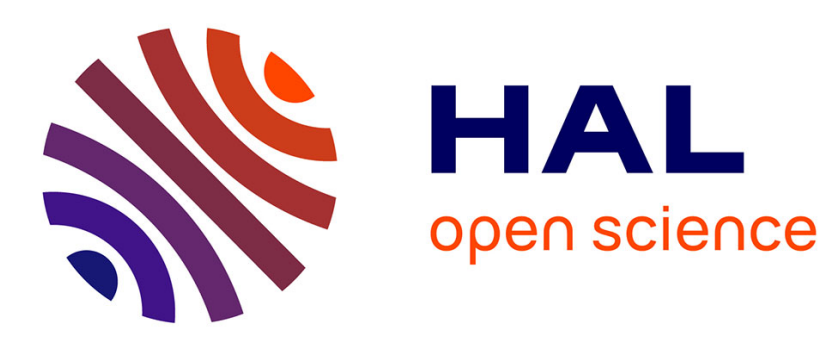

\title{
Effet du gaz carbonique et de la culture en climat artificiel sur la croissance et le rendement d'un blé d'hiver
}

Laurette Combe

\section{To cite this version:}

Laurette Combe. Effet du gaz carbonique et de la culture en climat artificiel sur la croissance et le rendement d'un blé d'hiver. Agronomie, 1981, 1 (3), pp.177-186. hal-00884240

\author{
HAL Id: hal-00884240 \\ https://hal.science/hal-00884240
}

Submitted on 1 Jan 1981

HAL is a multi-disciplinary open access archive for the deposit and dissemination of scientific research documents, whether they are published or not. The documents may come from teaching and research institutions in France or abroad, or from public or private research centers.
L'archive ouverte pluridisciplinaire HAL, est destinée au dépôt et à la diffusion de documents scientifiques de niveau recherche, publiés ou non, émanant des établissements d'enseignement et de recherche français ou étrangers, des laboratoires publics ou privés. 


\title{
Effet du gaz carbonique et de la culture en climat artificiel sur la croissance et le rendement d'un blé d'hiver
}

\author{
Laurette COMBE \\ Station de Bioclimatologie, I.N.R.A., Route de St Cyr, 78000 Versailles
}

RESUME

Blé,

Fumure carbonée,

Climat artificiel,

Croissance,

Rendement.

\begin{abstract}
Une fumure carbonée (à $950 \mathrm{vpm}$ de $\mathrm{CO}_{2}$ ) a été appliquée pendant 4 semaines, soit pendant la période (1) du développement floral, soit pendant la période (2) de la croissance des grains, sur des plantes de Blé cultivées en pots et transférées en enceinte. Leur croissance a été comparée à celle de plantes transférées en enceinte sans fumure carbonée (à $350 \mathrm{vpm}$ de $\mathrm{CO}_{2}$ ) pendant les mêmes périodes. La fumure carbonée augmente la production totale de matière sèche de la partie aérienne (fig. 2 et 3 ) et accroît le rendement en grains. Dans la période (1) la fumure carbonée agit un peu sur toutes les composantes du rendement mais surtout sur le nombre de grains par épi et améliore le rendement en grains de 00 p. 100 (tabl. 3). Dans la période (2), la fumure carbonée améliore le rendement de 40 p. 100 en multipliant le nombre de grains par épi, sans provoquer de diminution importante du poids de 1000 grains (tabl. 4).

De plus, la croissance de plantes restées à l'extérieur a été comparée avec celle de plantes transférées pendant 4 ou 8 semaines dans des enceintes à climat artificiel. Le climat des enceintes favorise l'élongation des tiges pendant la période (1) (fig. 4) et augmente le tallage et le nombre d'épis par pied surtout pendant la période (2) (tabl. 6).
\end{abstract}

\section{SUMMARY}

Wheat,

Carbon dioxide enrichment,

Air conditioned cultivation,

Growth,

Yield.
Effect of carbon dioxide and culture under artificial climate on the growth and yield of a winter wheat

A carbonaceous supply (at $950 \mathrm{vpm}$ of $\mathrm{CO}_{2}$ ) was made during 4 weeks either during the period (1) of flower development or during the period (2) of the grain growth on wheat seedlings grown in pots and removed to chambers. Their growth has been compared to that of seedlings removed to chambers without carbonaceous supply (at $350 \mathrm{vpm}$ of $\mathrm{CO}_{2}$ ) during the same periods. Carbonaceous supply increases the total production of dry matter of the aerial part (fig. 2 and 3 ) and the grain yield. During period (1) carbonaceous supply acts a little on all the yield components but mainly on the grain number per ear and improves the grain yield of 30 p. 100 (tabl. 3). During period (2) carbonaceous supply improves the yield of 40 p. 100 by multiplying the grain number per ear without inducing an important decrease of the weight of 10000 grains (tabl. 4). Moreover the growth of plants remained outside has been compared with that of plants transferred during 4 or 8 weeks in chambers with artificial climate. The climate of the chambers contributes to the elongation of the stems during period (1) (fig. 4) and increases tillering and the ear number per stalk especially during period (2) (table 6).

\section{INTRODUCTION}

L'activité photosynthétique des plantes est à la source de toute biomasse. Les études sur la photosynthèse des feuilles ont montré que les principaux facteurs d'action sont l'éclairement et la teneur en gaz carbonique de l'air (RABINOWITCH, 1951 ; CHARTIER, 1966) ; la température, l'alimentation hydrique et minérale, etc... interviennent aussi.

L'activité photosynthétique d'une plante est déterminante pour sa production; cependant certains travaux soulignent l'absence de corrélation entre la capacité photosynthétique de l'unité de surface de feuille et la production agricole d'une plante. C'est le cas chez le blé où les variétés modernes (à fort rendement) ont une photosynthèse par unité de surface plus faible (mais ont souvent une surface assimilatrice plus grande) que les variétés (peu productives) primitives (Evans \& DUnSTONE, 1970 ; Evans, 1975).

La production agricole peut être limitée par d'autres phénomènes que la photosynthèse, comme par exemple la répartition des produits de la photosynthèse (les assimilats) ou la capacité d'accumulation de ces produits par les organes en croissance (WARDLAW, 1976). C'est-à-dire que les relations entre les sources (organes producteurs d'assimilats) et les puits (organes demandeurs d'assimilats) sont aussi déterminantes que l'assimilation proprement dite pour le rendement agricole final d'une culture (TOLLENAAR, 1977).

Il a été montré d'ailleurs que le niveau d'assimilation 
lui-même agissait sur la distribution des assimilats à l'intérieur de la plante (RYLE \& POWELL, 1976). Pour savoir si la photosynthèse est ou non un facteur limitant de la production, il faut vérifier qu'une augmentation de photosynthèse nette par plante entraîne un accroissement de la production (HARDMAN \& BRUN, 1971).

La fumure carbonée, c'est-à-dire l'enrichissement de l'air en gaz carbonique, permet d'augmenter la photosynthèse des plantes dites en $\mathrm{C} 3$ telles que le blé. Le travail de KRENZER (1974) sur 2 variétés américaines de blé a déjà montré qu'une fumure carbonée appliquée pendant la phase de croissance végétative n'avait pas d'effet sur la production de grains. Par contre, appliquée pendant le développement floral, la fumure carbonée augmente le nombre de grains et appliquée pendant la croissance des grains, elle accroît le poids de 1000 grains. Dans les 2 cas, l'enrichissement en $\mathrm{CO}_{2}$ permet d'accroître la production de grains.

Les expériences décrites ici avaient pour but de vérifier les résultats de KRENZER sur une variété européenne de blé d'hiver et de préciser l'action de la fumure carbonée sur la croissance et le développement de la plante entière et non pas seulement sur le rendement final en grains. De plus, comme les plantes étaient cultivées temporairement en enceinte à atmosphère artificielle pour procéder aux fumures carbonées, la comparaison de la croissance en climat artificiel et en climat extérieur s'avérait nécessaire.

\section{CONDUITE DES EXPÉRIENCES}

\subsection{Matériel végétal}

Les expériences ont été réalisées avec la variété demi-naine de blé d'hiver «Maris Hobbit» d'origine anglaise, considérée aujourd'hui comme l'une des plus productives $(\mathrm{M}$ Hobbit $=111$ p. 100 de Champlein, cv. de référence) dans le nord de la France.

\subsection{Mise en culture}

Après germination sur papier filtre imbibé d'eau pendant 3 jours, les semences ont été placées dans des pots remplis de terre de $40 \mathrm{~cm}$ de profondeur et de $20 \mathrm{~cm} \times 25 \mathrm{~cm}$ de surface. La mise en place des semences germées a eu lieu le 30 novembre 1978 à Versailles. Les pots étaient posés dehors dans un trou de $0,4 \mathrm{~m}$ de profondeur de façon à reconstituer une petite parcelle de $2 \mathrm{~m} \times 1 \mathrm{~m}$ entourée de gazon.

Après la levée, les pots ont été éclaircis de façon à garder au plus 12 plantes par pot ; mais les gelées de l'hiver ayant détruit un grand nombre de plantules, certains pots n'avaient même pas 12 plantes.

\subsection{Traitements}

Les plantes sont toutes restées à l'extérieur jusqu'au 23 mai 1979 (soit $174 \mathrm{j}$ après la germination, ou une somme de température de $966^{\circ} \mathrm{j}$. au-dessus de $0^{\circ} \mathrm{C}$ ). Pour soumettre les plantes à une fumure carbonée, les pots ont été transférés dans des enceintes de culture à atmosphère artificielle où la teneur en $\mathrm{CO}_{2}$ de l'air pouvait être maintenue à un niveau donné.
Ces enceintes ont une surface au sol de $0,5 \mathrm{~m}^{2}$, la hauteur utile est de $2,45 \mathrm{~m}$; l'éclairement est obtenu avec 6 lampes HQI (aux halogènes métalliques) dont le spectre d'émission mesuré en place couvre tout le visible avec une pointe autour de $530 \mathrm{~nm}$. L'atmosphère des enceintes est régulée en circuit fermé : des échangeurs latéraux permettent de maintenir la température de l'air au niveau de la consigne (une température de jour, une température de nuit). Le point de rosée n'est pas actuellement régulé.

De même, la teneur en gaz carbonique de l'air est analysée puis réajustée en permanence grâce à un détecteur de seuil commandant une injection de $\mathrm{CO}_{2}$ (quand il y a photosynthèse) ou une élimination de $\mathrm{CO}_{2}$ (quand il y a respiration). La teneur en gaz carbonique était de $950 \pm 50 \mathrm{vpm}$ dans l'enceinte avec fumure carbonée. Dans le même temps, d'autres pots étaient placés comme témoins dans une enceinte identique mais sans fumure carbonée, c'est-à-dire à $350 \pm 50 \mathrm{vpm}$, ce qui est légèrement supérieur à la teneur normale de l'air en $\mathrm{CO}_{2}$. Chaque enceinte contenait 8 pots.

Autant que possible, le climat dans les enceintes se rapprochait du climat extérieur. La durée du jour, les températures diurne et nocturne étaient réglées chaque semaine à un niveau donné. La durée du jour — qui varie peu (15 h 30-16 h 06) pendant la période considérée - était la même, à 7 min près, qu'à l'extérieur ; mais l'éclairement était 1,5 à 2 fois plus élevé dans les enceintes qu'à l'extérieur (fig. 1a). Les températures diurne et nocturne d'une semaine étaient calculées à partir des relevés de température extérieure de la semaine précédente. Toutefois, la température moyenne dans les enceintes a été un peu plus élevée qu'à l'extérieur pendant toute la durée de l'expérimentation (fig. 1b).

Le tableau 1 résume les conditions des différents traitements. 2 traitements de fumure carbonée ont été appliqués sur 2 lots différents de 8 pots chacun. Le premier traitement a eu lieu dans la phase de différenciation florale, période (1) du 23 mai (pleine montaison) au 20 juin (fin de l'anthèse) (lot TR1). Le second traitement a eu lieu dans la phase de croissance des grains, période (2) du 20 juin (fin de l'anthèse) au 18 juillet (récolte à maturité) (lot TR2). Chaque traitement a duré 4 semaines.

Quatre pots sont restés dans l'enceinte-témoin pendant les 8 semaines de traitement (lot TE) ; 4 pots, mis dans l'enceinte témoin au début du traitement, en ont été retirés en même temps que les pots TR1 (lot TE1). De même, 4 pots ont été placés dans l'enceinte-témoin pendant la seconde période de traitement (lot TE2). 12 pots sont restés à l'extérieur pendant tout le temps de l'expérience (lot EXT).

Les plantes restées à l'extérieur n'ont reçu que l'eau de pluie, soit $57 \mathrm{~mm}$ d'eau pour la période (1) et $21 \mathrm{~mm}$ pour la période (2) (il y a eu 24 jours sans pluie sur cette période de 28 jours). Au contraire, les plantes mises en enceintes ont bénéficié d'une irrigation quotidienne plus que suffisante.

\subsection{Mesures}

Des prélèvements de 6 à 12 plantes ont été effectués chaque semaine sur les lots EXT, TR1 et TR2 et chaque quinzaine sur les lots TE1, TE2 et TE. Des mesures de longueur des tiges, de poids de matière fraîche et de matière sèche ont été faites, plante par plante et organe par organe, pour la partie aérienne (feuilles, tiges, épis).

A la récolte, l'ensemble des plantes restantes dans chaque lot a été compté, pesé, séché, etc... de façon à établir les différentes composantes du rendement final. 

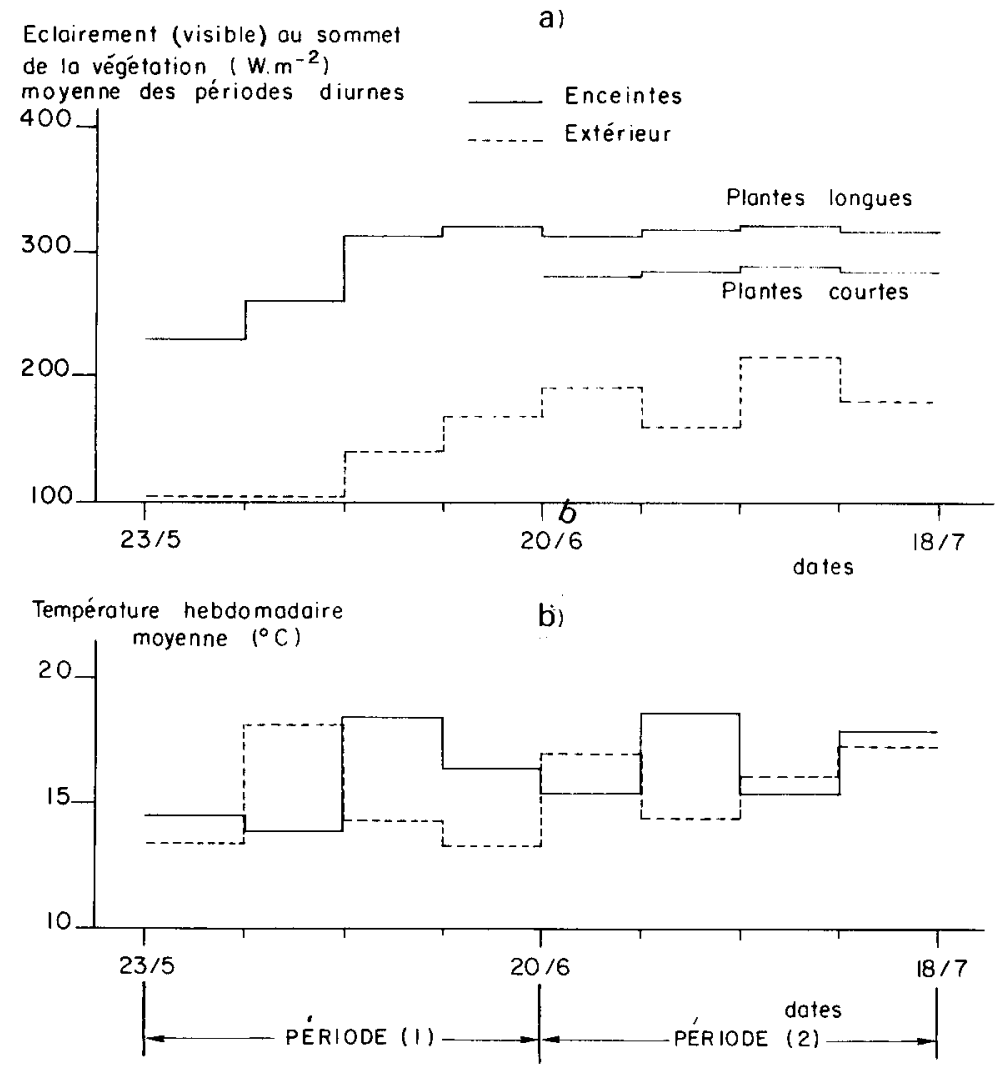

Figure 1

Comparaison du climat dans les enceintes avec le climat extérieur.

Climate in the field and in air conditioned units : a) visible irradiance at the top of the crop; mean of daylight periods, — air conditioned units, - . . field. b) mean weekly temperature.

TABLEAU 1

Résumé des conditions des différents traitements Summary of the different treatments conditions

\begin{tabular}{|c|c|c|c|c|c|c|c|}
\hline \multicolumn{2}{|c|}{$\begin{array}{l}\text { Nom du traitement } \\
\text { et nombre de pots }\end{array}$} & $\begin{array}{c}\text { EXT } \\
12\end{array}$ & $\begin{array}{c}\mathrm{TE} \\
4\end{array}$ & $\begin{array}{c}\text { TE1 } \\
4\end{array}$ & $\begin{array}{c}\text { TR1 } \\
8\end{array}$ & $\begin{array}{c}\text { TE2 } \\
4\end{array}$ & $\begin{array}{c}\text { TR2 } \\
8\end{array}$ \\
\hline \multicolumn{8}{|c|}{ Conditions de culture: } \\
\hline en enceinte & $\begin{array}{l}\text { pendant } \\
\text { période (1) }\end{array}$ & non & oui & oui & oui & non & non \\
\hline artificielle & $\begin{array}{l}\text { pendant } \\
\text { période (2) }\end{array}$ & non & oui & non & non & oui & $o u i$ \\
\hline avec fumure & $\begin{array}{l}\text { pendant } \\
\text { période (1) }\end{array}$ & non & non & non & oui & non & non \\
\hline carbonée & $\begin{array}{l}\text { pendant } \\
\text { période (2) }\end{array}$ & non & non & non & non & non & oui \\
\hline
\end{tabular}

- à l'extérieur (pas en enceinte) : pas d'apport d'eau supplémentaire (seulement la pluie), éclairement naturel, fortes fluctuations de température.

- en enceinte au contraire : irrigation quotidienne, éclairement artificiel plus élevé qu'à l'extérieur (constant), températures de jour et de nuit constantes pendant une semaine (température moyenne légèrement plus élevée qu'à l'extérieur).

- pas de fumure carbonée $=350 \pm 50 \mathrm{vpm} \mathrm{CO} \mathrm{CO}_{2}$
- fumure carbonée $=950 \pm 50 \mathrm{vpm} \mathrm{CO}_{2}$

- période (1) du $23 / 5$ au $20 / 6$.

- période (2) du $20 / 6$ au $18 / 7$.

- in the field (not air conditioned) : no additional water supply (only rainfall), natural irradiance, marked temperature changes.

- in air conditioned units : daily watering, stronger (constant) artificial irradiance, constant day and night temperature for one week (mean temperature sightly higher than in the field).

- no high $\mathrm{CO}_{2}=350 \pm 50 \mathrm{vpm} \mathrm{CO}_{2}$.

- high $\mathrm{CO}_{2}=950 \pm 50 \mathrm{vpm} \mathrm{CO}_{2}$.

- period (1) : $23 / 5$ to $20 / 6$.

- period (2) : $20 / 6$ to $18 / 7$. 


\section{RÉSULTATS : ANALYSE ET DISCUSSION}

Pour plus de clarté, les résultats sont regroupés en 2 parties : dans la $1^{\text {ère }}$, sont exposés les effets de la fumure carbonée sur la croissance et la répartition de la matière sèche dans la plante de blé. Les effets de la culture en climat artificiel sont décrits en seconde partie.

\subsection{Effet des fumures carbonées}

\subsection{La croissance}

\subsection{Croissance des tiges}

La fumure carbonée n'a pas eu d'effet sur l'élongation des tiges ni en période (1) (pour TE1, $1=68,1 \mathrm{~cm}$ et pour TR1, $1=67,5 \mathrm{~cm}$; différence non significative), ni en période (2) (pour TE2, $1=50,9 \mathrm{~cm}$ et pour TR2, $1=52,2 \mathrm{~cm}$; différence non significative).

Par contre, la masse (matière sèche) de tige rapportée à l'unité de longueur est modifiée en période (2) par l'application de la fumure carbonée : pour TE1 $(\mathrm{MS} / \mathrm{l})=1,5 \mathrm{mg} \mathrm{cm}^{-1}$ et pour TR1 $(\mathrm{MS} / \mathrm{l})=1,3 \mathrm{mg} \mathrm{cm}^{-1}$ différence non significative ; pour TE2 $(\mathrm{MS} / \mathrm{l})=1,1 \mathrm{mg} \mathrm{cm}^{-1}$ et pour TR2
$(\mathrm{MS} / \mathrm{l})=2,2 \mathrm{mg} \mathrm{cm} \mathrm{cm}^{-1}$, différence significative au seuil 0,001 .

L'accumulation apparente de matière sèche dans les tiges des plantes « enrichies en $\mathrm{CO}_{2}$ » pendant la période (2) est en fait une moindre perte de matière sèche des tiges (au profit des épis) lorsque la photosynthèse est renforcée pendant la période de redistribution de la matière sèche dans la plante (voir plus loin $\$ 3.1 .2$.).

\subsection{Croissance de la partie aérienne}

La fumure carbonée (précoce ou tardive) augmente la croissance globale en matière sèche par pied (fig. 2 et 3 ). Les plantes traitées pendant la période (1) ont leur croissance augmentée immédiatement et la différence avec les les plantes des 2 lots TE1 et TR1 sont replacées à l'extérieur. La fumure carbonée pendant la période (2) sur des plantes qui étaient auparavant à l'extérieur, non seulement empêche le ralentissement de croissance constaté dans le lot TE2, mais encore augmente la croissance en matière sèche par pied du lot TR2, sans atteindre le niveau du lot TR1 cependant.

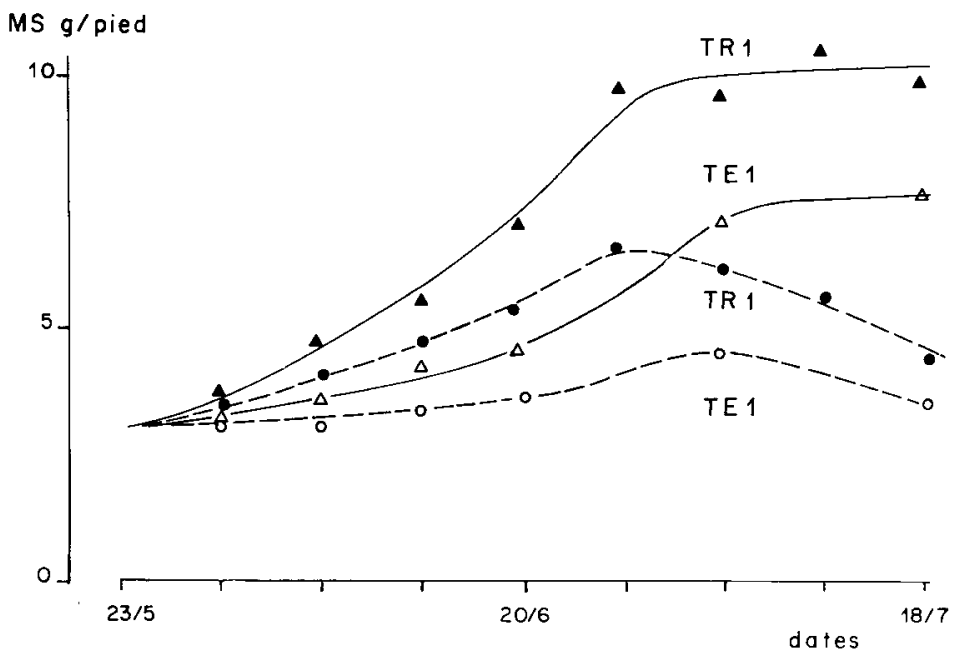

Figure 2

Effet de la fumure carbonée précoce sur la croissance en matière sèche de la partie aérienne et sur la répartition de la matière sèche entre la paille et les épis.

Effect of early high $\mathrm{CO}_{2}$ on dry matter growth of the aerial part and on dry matter distribution between straw and ears. - aerial parts (straw + ears) . . - - straw (only); ordinate: d.m. g/plant.

Figure 3

Effet de la fumure carbonée tardive sur la croissance en matière sèche de la partie aérienne et sur la répartition de la matière sèche entre la paille et les épis.

Effect of late high $\mathrm{CO}_{2}$ on dry matter growth of the aerial part and on dry matter distribution straw and ears : aerial parts (straw + ears), - . - - straw (only): d.m. g/plant.

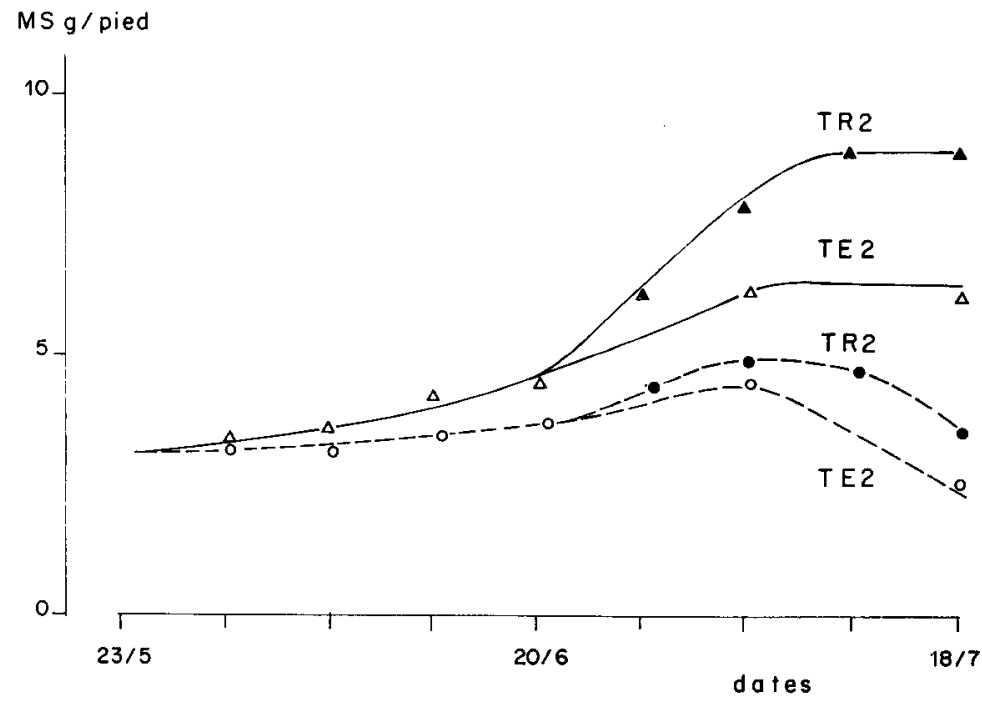




\subsection{Répartition de la matière sèche entre la paille et les épis}

Les figures 2 et 3 montrent que la croissance (en matière sèche) globale de la partie aérienne s'arrête avant la maturité de récolte. A partir du moment où la partie aérienne atteint son maximum de poids, une redistribution de la matière sèche se produit à l'intérieur de la plante : la tige et les feuilles ( $=$ la paille) perdent du poids au profit des épis.

Cette redistribution de la matière sèche vers les épis est plus précoce pour les plantes ayant eu une fumure carbonée

\section{TABLEAU 2}

Effet de la fumure carbonée sur la composition du couvert végétal le 20.6.79, à la fin de la première période de traitement

Effect of high $\mathrm{CO}_{2}$ on the composition of the aerial part of crops at the end of the first treatment period (6.20.79)

\begin{tabular}{lcc}
\hline \hline & TE1 & TR1 \\
\hline $\begin{array}{l}\text { Nombre de pieds/m² (number plants/ } \\
\mathrm{m}^{2} \text { ) }\end{array}$ & 210 & 210 \\
$\begin{array}{l}\text { Nombre de talles/pied (number of til- } \\
\text { lers/plant) }\end{array}$ & 3,86 & $4,33 *$ \\
$\begin{array}{l}\text { Nombre d'épis/pied (number of ears/ } \\
\text { plant) }\end{array}$ & 2,50 & 2,55 \\
$\begin{array}{l}\text { Poids de matière sèche/épi (mg) (dry mat- } \\
\text { ter weight/ear (mg)) }\end{array}$ & 403 & $608 *$ \\
$\begin{array}{l}\text { Poids d'épis/m (g de m. sèche) (ear } \\
\text { weight } / \mathrm{m}^{2} \text { (g of dry matter)) }\end{array}$ & 212 & $326 * *$ \\
$\begin{array}{l}\text { Poids total/m } \\
\text { weight } / \mathrm{m}^{2} \text { ) }\end{array}$ & $\mathrm{g}$ de m. sèche) (total \\
$\begin{array}{l}\text { Poids d'épis/poids total (ear weight/total } \\
\text { weight) }\end{array}$ & 964 & $1424 * *$ \\
$\begin{array}{l}\text { Pourcentage d'eau dans la plante (water } \\
\text { content in the plant (p. 100)) }\end{array}$ & $62 \%$ & $70 \%$ \\
\hline
\end{tabular}

* Différence avec le lot TE1 significative au seuil de $5 \%$.

** Différence avec le lot TE1 significative au seuil de $1 \%$. pendant la période (1) (lot TR1) que pour les plantes témoins (lot TE1). Elle est, au contraire, plus tardive pour les plantes ayant eu une fumure carbonée pendant la période (2) (lot TR2) que pour les témoins correspondants (lot TE2). Nous avons vu plus haut que cela se traduisait par une plus grande masse de matière sèche par unité de longueur dans les tiges.

\subsection{Composition du couvert végétal à la fin de la période (1)}

A la fin de la période (1), la fumure carbonée sur les plantes cultivées en enceinte permet d'obtenir un plus grand nombre de talles $(+12$ p. 100, différence significative au seuil 0,05$)$ mais le même nombre d'épis par pied $(+2$ p. 100 non significatif), mais ceux-ci sont plus lourds $(+51$ p. 100) que les épis des plantes témoins (voir tableau 2). La production d'épis par plante est donc plus grande $(+54$ p. 100) pour le lot TR1 que pour le lot TE1. La production totale de matière sèche aérienne est également plus forte pour les plantes cultivées en enceinte avec fumure carbonée (lot TR1) que pour les autres (lot TE1). Il est à noter que la répartition de la matière sèche dans la partie aérienne, entre les épis d'une part et les feuilles et les tiges d'autre part, n'est pas significativement différente dans les deux lots.

\subsection{Les composantes du rendement à la récolte \\ 3.141. Effet de la fumure carbonée pendant la période (1)}

La comparaison des tableaux 2 et 3 permet de constater que la fumure carbonée précoce n'a finalement pas d'effet sur le nombre de talles présentes à la récolte. Elle n'a pas non plus d'effet significatif sur le nombre d'épis par plante. L'augmentation $(+12$ p. 100) du nombre de grains par épi est significative au seuil de 0,05 (voir tableau 3 ). Le poids de 1000 grains n'est pas significativement plus élevé pour le traitement fumure carbonée. La combinaison d'effets peu ou pas significatifs aboutit cependant à une récolte en grains significativement (seuil de 0,025$)$ supérieure $(+29$ p. 100) pour les plantes avec fumure carbonée ; il en est de même

\section{TABLEAU 3}

Effet d'une fumure carbonée précoce sur les composantes de la production à la récolte, le 18.7.79 Effect of early high $\mathrm{CO}_{2}$ on yield components upon harvest (7.8.79)

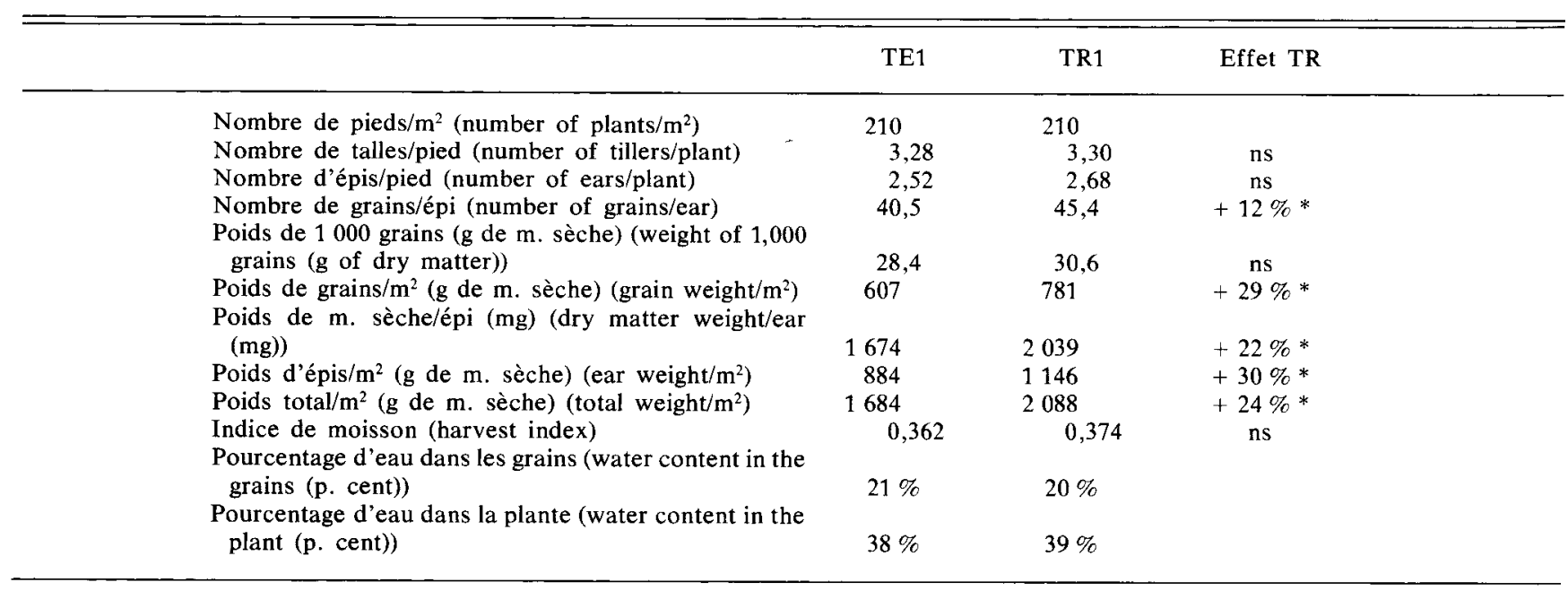


pour la production de matière sèche aérienne. L'indice de moisson n'est pas significativement différent entre les 2 traitements TE1 et TR1.

\subsection{Effet de la fumure carbonée pendant la période (2)}

La fumure carbonée dans la période (2) accroît fortement (+ 48 p. 100) le nombre de grains par épi, sans diminution significative du poids de 1000 grains (tabl. 4). Ce qui permet une augmentation de la récolte en grains de 39 p. 100. L'augmentation de la biomasse aérienne totale est proportionnellement la même que l'augmentation du poids de grains: l'indice de moisson n'est pas différent entre les 2 traitements TR2 et TR2.

\subsection{Discussion}

Nos expériences montrent que la production totale de matière sèche de la partie aérienne est accrue aussi bien par une fumure carbonée précoce (période 1) que par une fumure tardive (période 2).

La fumure carbonée pendant la période (1) entraîne une augmentation du nombre de grains par épi sans augmenter significativement le poids de 1000 grains. Ce résultat est en accord avec ceux de KRENZER (1974).

Par contre, la fumure carbonée pendant la période (2) provoque encore une augmentation (très forte) du nombre de grains par épi sans entraîner une diminution significative du poids de 1000 grains. Le grossissement des grains n'a pas été accru par la fumure carbonée pendant la période (2) comme l'avait trouvé KRENZER (1974) avec d'autres variétés de blé. Le phénomène de multiplication du nombre de grains après l'anthèse par la fumure carbonée correspond probablement à une diminution du phénomène d'avortement : le nombre de grains qui avortent ou qui ne grossissent pas est diminué par la fumure carbonée. De ce point de vue, on peut considérer que le grossissement des grains a été favorisé par la fumure carbonée pendant la période (2), comme le montre KRENZER (1974).

L'augmentation totale de la production de matière sèche par les fumures carbonées s'accompagne d'une augmentation proportionnelle de la production de grains. Autrement dit, l'indice de moisson n'a pas été modifié par la fumure carbonée dans nos expériences, contrairement à ce que trouve par exemple GIFFORD (1977).

Le brusque changement de climat (provoqué par la mise èn enceinte), dans les expériences présentées ici, a perturbé le développement des plantes, il est donc difficile de comparer nos résultats avec ceux d'autres auteurs obtenus avec d'autres variétés de blé dans d'autres conditions climatiques.

\subsection{Effet de la culture en climat artificiel}

\subsection{Croissance des tiges}

Les plantes qui ont poussé à l'extérieur pendant la période (1) (23 mai-20 juin) (lots EXT, TE2 et TR2) et celles qui ont été placées en enceinte durant la même période (lots TE, TE1 et TR1) ont des croissances en hauteur nettement différentes (fig. 4). L'essentiel de l'élongation de la tige a eu lieu pendant la période (1). L'élongation du dernier entrenoud s'est un peu poursuivie pendant la période (2), surtout pour les plantes mises dans les enceintes.

La culture en enceinte a permis une plus forte $(+60$ p. 100) élongation des tiges et particulièrement du dernier entre-nœud: le rapport des longueurs (dernier entre-noud/tige) est de 0,38 pour le lot EXT et de 0,46 pour le lot TE ; la différence est significative au seuil de 0,001 .

Il est à noter un effet significatif au seuil de 0,05 de la culture en enceinte (lot TE) par rapport aux plantes extérieures sur la densité linéaire des tiges : $1,5 \mathrm{mg} \mathrm{cm}^{-1}$ pour EXT et $2,0 \mathrm{mg} \mathrm{cm}^{-1}$ pour TE. La culture en climat artificiel (fort éclairement) diminue donc, elle aussi, la redistribution de matière sèche des tiges vers les épis.

Effet d'une fumure carbonée tardive sur les composantes de la production à la récolte, le 18.7.79 Effect of late $\mathrm{CO}_{2}$ on yield components upon harvest (7.18.79)

\begin{tabular}{|c|c|c|c|}
\hline & TE2 & TR2 & Effet TR \\
\hline $\begin{array}{l}\text { Nombre de pieds } / \mathrm{m}^{2} \text { (number of plants } / \mathrm{m}^{2} \text { ) } \\
\text { Nombre de talles/pied (number of tillers/plant) } \\
\text { Nombre d'épis/pied (number of ears/plant) } \\
\text { Nombre de grains/épi (number of grains/ear) } \\
\text { Poids de } 1000 \text { grains (g de } \mathrm{m} \text {. sèche) (weight of } 1,000 \\
\text { grains (g of dry matter)) } \\
\text { Poids de grains } / \mathrm{m}^{2} \text { ( } \mathrm{g} \text { de } \mathrm{m} \text {. sèche) (grain weight } / \mathrm{m}^{2} \text { (g } \\
\text { of dry matter)) } \\
\text { Poids de } \mathrm{m} \text {. sèche/épi ( } \mathrm{mg} \text { ) (dry matter weight/ear } \\
\text { (mg)) } \\
\text { Poids d'épis } / \mathrm{m}^{2} \text { (g de } \mathrm{m} \text {. sèche) (ear weight } / \mathrm{m}^{2} \text { ) } \\
\text { Poids total } / \mathrm{m}^{2} \text { (g de } \mathrm{m} \text {. sèche) (total weight } / \mathrm{m}^{2} \text { ) } \\
\text { Indice de moisson (harvest index) } \\
\text { Pourcentage d'eau dans les grains (water content in the } \\
\text { grains) } \\
\text { Pourcentage d'eau dans la plante (water content in the } \\
\text { plant) }\end{array}$ & $\begin{array}{c}215 \\
3,04 \\
1,89 \\
32,1 \\
47,5 \\
622 \\
1919 \\
783 \\
1366 \\
0,455 \\
24 \% \\
50 \%\end{array}$ & $\begin{array}{c}210 \\
3,16 \\
1,98 \\
47,4 \\
\\
44,2 \\
\\
867 \\
2681 \\
1110 \\
1866 \\
0,467 \\
\\
23 \% \\
\\
54 \%\end{array}$ & $\begin{array}{c}\mathrm{ns} \\
\mathrm{ns} \\
+48 \% \%^{* * *} \\
\mathrm{~ns} \\
+39 \% \%^{* *} \\
+40 \% \%^{* *} \\
+41 \% \%^{* *} \\
+37 \% \%^{* *} \\
\mathrm{~ns}\end{array}$ \\
\hline
\end{tabular}

** Différence avec le lot TE2 significative au seuil de $1 \%$.

*** Différence avec le lot TE2 significative au seuil de $1 \%$

ns Différence avec le lot TE2 non significative. 


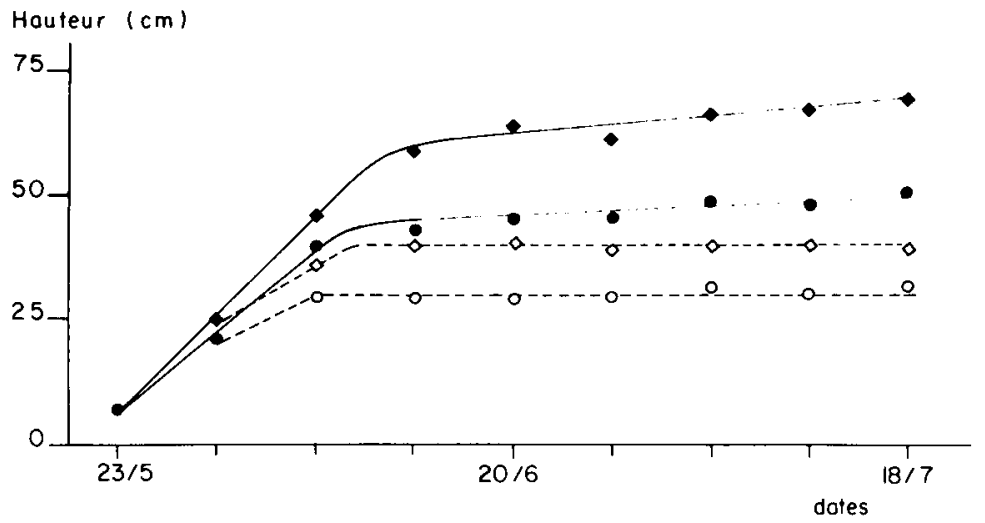

Figure 4

Comparaison des élongations des tiges des plantes restées à l'extérieur avec les plantes cultivées en enceinte pendant la période (1) de développement floral.

Stalk elongation in field crop and in air conditioned unit crop during period (1) floral development

Longueur totale de la tige (total length of the stalk);

-... longueur de la tige sans le dernier entre-noud (lenght of the stalk minus the last internode); plantes cultivées en enceinte (air conditioned unit crop) ;

- plantes restées a l'extérieur (field crop).
Figure 5

Effet de la culture en enceinte sur la croissance en matière sèche de la partie aérienne.

Effect of cultivation in air conditioned unit on dry matter growth of the aerial part; ordinate: d.m. g/plant.

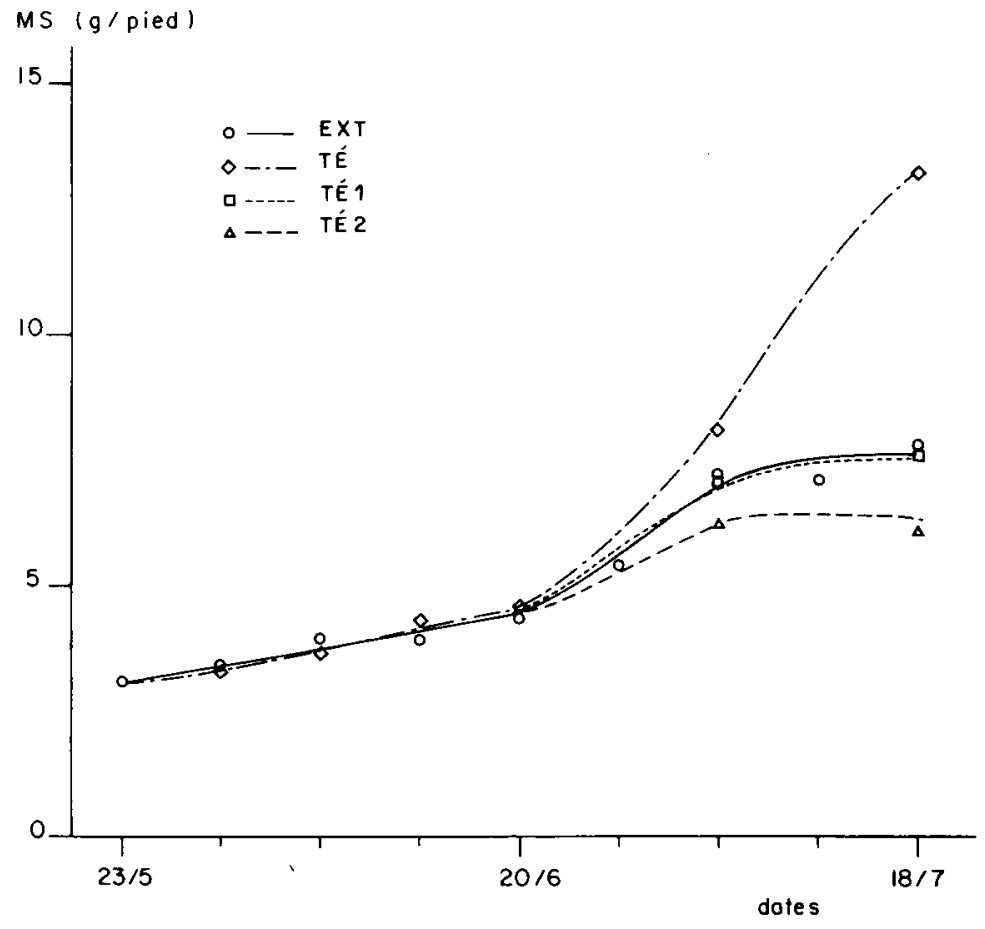

TABLEAU 5

Effet de la culture en enceinte sur la composition du couvert végétal le 20.6.79, à la fin de la première période de traitement

Effect of air conditioned cultivation on the composition of the aerial part of crops at the end of the first treatment period (6.20.79)

\begin{tabular}{|c|c|c|}
\hline & EXT & TE1 \\
\hline \multicolumn{3}{|c|}{$\begin{array}{c}\text { Nombre de pieds } / \mathrm{m}^{2} \text { (number of } \\
\text { plants } / \mathrm{m}^{2} \text { ) }\end{array}$} \\
\hline $\begin{array}{c}\text { Nombre de talles/pied (number of } \\
\text { tillers/plant) }\end{array}$ & 3,07 & $3,86^{*}$ \\
\hline $\begin{array}{l}\text { Nombre d'épis/pied (number of } \\
\text { ears/plant) }\end{array}$ & 2,01 & $2,50 *$ \\
\hline $\begin{array}{l}\text { Poids de matière sèche/épi (mg) (dry mat- } \\
\text { ter weight/ear (mg)) }\end{array}$ & 518 & $403 *$ \\
\hline $\begin{array}{l}\text { Poids d'épis } / \mathrm{m}^{2} \text { ( } \mathrm{g} \text { de } \mathrm{m} \text {. sèche) (ear } \\
\text { weight } / \mathrm{m}^{2}(\mathrm{~g} \text { of dry matter)) }\end{array}$ & 219 & 212 \\
\hline $\begin{array}{l}\text { Poids total } / \mathrm{m}^{2} \text { (g de } \mathrm{m} \text {. sèche) (total } \\
\text { weight } / \mathrm{m}^{2} \text { ) }\end{array}$ & 920 & 964 \\
\hline $\begin{array}{l}\text { Poids d'épis/poids total (ear weight/total } \\
\text { weight) }\end{array}$ & 0,238 & 0,220 \\
\hline $\begin{array}{l}\text { Pourcentage d'eau dans la plante (water } \\
\text { content in the plant) }\end{array}$ & $55 \%$ & $62 \%$ \\
\hline
\end{tabular}

* Différence avec le lot EXT significative au seuil de $5 \%$.
Le tableau 5 montre que la croissance globale, pendant la période (1), des lots EXT et TE2 se décompose de façon différente. La culture en enceinte favorise le tallage ou empêche le dessèchement des jeunes tiges et augmente le nombre d'épis par plante. Sans fumure carbonée, les épis des plantes cultivées en enceinte sont plus légers ( -22 p. 100) que les épis des plantes restées à l'extérieur. Comme le nombre d'épis est plus grand, le poids d'épis par 
plante est sensiblement le même pour le lot TE1 et pour le lot EXT.

Par contre, la teneur en eau des plantes cultivées en enceinte est plus élevée que celle des plantes extérieures surtout en présence de fumure carbonée.

\subsection{Les composantes du rendement à la récolte}

L'accroissement du tallage constaté le 20/6 (tabl. 5) s'est maintenu pour les plantes (TE) qui sont restées dans les enceintes ; pour celles qui ont été retirées des enceintes en fin de période (1), par contre, les talles supplémentaires ont séché et avaient disparu, en fin de période (2), lors de la récolte (tabl. 6).

L'augmentation du nombre d'épis par plante s'est poursuivie pendant la période (2) pour les plantes restées en enceinte; pour celles qui ont été ôtées des enceintes ou mises dans les enceintes au 20/6, le nombre d'épis par plante n'a pas été modifié pendant la période (2).

Le nombre de grains par épi a été augmenté par la culture en enceinte pendant les 2 périodes $(+30$ p. 100) alors que la culture en enceinte, pendant la période (1) seule, n'accroît pas significativement le nombre de grains par épi et que la culture en enceinte, pendant la période (2) seule, diminue ( -15 p. 100) le nombre de grains par épi.

La multiplication du nombre de grains par épi dans le lot TE s'accompagne d'une diminution égale du poids de 1000 grains ( -30 p. 100). De même, dans le lot TE2, la diminution du nombre de grains par épi est à peu près compensée par l'augmentation du poids de 1000 grains $(+12$ p. 100). Les grains du lot TE1 sont les plus petits : le remplissage s'est moins bien fait chez les plantes remises à l'extérieur après avoir séjourné en enceinte que chez les plantes restées à l'extérieur.

Le rendement final en grains est de $101 \mathrm{dg} \mathrm{dm}^{-2}$ pour les plantes restées en enceinte contre $68 \mathrm{dg} \mathrm{dm}^{-2}$ pour celles restées à l'extérieur. Cette augmentation (significative au seuil de 5 p. 100) provient uniquement de l'augmentation du nombre d'épis par plante, mais les grains sont petits, ce qui n'est pas intéressant pour l'agriculture. Pour les 2 autres lots de plantes, la récolte est légèrement inférieure (61 et $62 \mathrm{dg} \mathrm{dm}^{-2}$ ) à celle des plantes extérieures, mais la différence n'est pas significative.

Les mêmes différences de production totale de matière sèche aérienne se produisent entre les lots. L'indice de moisson (poids de grains/poids de matière sèche aérienne) n'est pas significativement différent entre les lots EXT, TE et TE1 ; il est un peu plus élevé ( +12 p. 100) pour le lot TE2. Les plantes restées en enceinte pendant la seconde période ont une teneur en eau plus élevée que les autres.

\subsection{Discussion}

La comparaison d'une culture temporaire dans une atmosphère artificielle (en enceinte) avec une culture à l'extérieur permet de dégager plusieurs enseignements de ces expériences sur le blé.

La culture poursuivie en enceinte pendant les périodes (1) et (2) provoque la multiplication des «tardillons " (épis développés tardivement). C'est un phénomène connu (AURIAU, comm. pers.) que l'on constate lorsque les plantes disposent d'eau en abondance et de lumière à suffisance. En particulier, l'éclairement élevé des enceintes a sans doute diminué l'effet de compétition pour la lumière (BALDY, 1974). Cette multiplication du nombre des épis s'accompagne d'une augmentation du nombre de grains par épi et aboutit à une forte multiplication des grains, mais ceux-ci ne sont pas très gros.

La culture en enceinte pendant la période (1) seule, suivie d'une remise en culture à l'extérieur, entraîne aussi une multiplication du nombre d'épis formés et du nombre de grains par épi (augmentation plus faible toutefois que dans le cas précédent) et les grains ne grossissent pas beaucoup non plus. Ici aussi, probablement, l'abondance d'eau pendant la culture en enceinte est la principale cause des différences avec les plantes restées à l'extérieur (non irriguées).

La mise en enceinte de culture artificielle pendant la période (2) a plutôt un effet négatif $(-20 \mathrm{p}$. 100) sur la croissance globale de la partie aérienne, mais la production

TABLEAU 6

Effet de la culture en enceinte sur les composantes de la production à la récolte, le 18.7.79 (fin de la seconde période de traitement)

Effect of air conditioned cultivation on yield components upon harvest (7.18.79)

\begin{tabular}{|c|c|c|c|c|}
\hline & $\mathrm{EXT}$ & TE & TE1 & TE2 \\
\hline Nombre de pieds $/ \mathrm{m}^{2}$ (number of plants $/ \mathrm{m}^{2}$ ) & 210 & 205 & 210 & 215 \\
\hline Nombre de talles/pied (number of tillers/plant) & 3,21 & 3,83 & 3,28 & 3,04 \\
\hline Nombre d'épis/pied (number of ears/plant) & 2,02 & $3,37 *$ & 2,52 & 1,89 \\
\hline Nombre de grains/épi (number of grains/ear) & 37,6 & $48,9 * *$ & 40,5 & 32,1 \\
\hline $\begin{array}{l}\text { Poids de } 1000 \text { grains (g de } \mathrm{m} \text {. sèche) (weight of } 1000 \\
\text { grains (g of dry matter)) }\end{array}$ & 42,4 & $30,0 * * *$ & 28,4 & 47,5 \\
\hline Poids de grains $/ \mathrm{m}^{2}\left(\mathrm{~g}\right.$ de $\mathrm{m}$. sèche) (grain weight $/ \mathrm{m}^{2}$ ) & 676 & $1009 *$ & 607 & 622 \\
\hline Poids de m. sèche/épi (mg) (dry matter weight/ear) & 2146 & 2113 & 1674 & 1919 \\
\hline Poids d'épis $/ \mathrm{m}^{2}$ ( $\mathrm{g}$ de $\mathrm{m}$. sèche) (ear weight $/ \mathrm{m}^{2}$ ) & 910 & 1454 & 884 & 783 \\
\hline Poids total $/ \mathrm{m}^{2}$ (g de $\mathrm{m}$. sèche) (total weight $/ \mathrm{m}^{2}$ ) & 1707 & 2694 * & 1684 & 1366 \\
\hline Indice de moisson (harvest indice) & 0,396 & 0,374 & 0,362 & $0,455 *$ \\
\hline $\begin{array}{l}\text { Pourcentage d'eau dans les grains (water content in the } \\
\text { grains) }\end{array}$ & $17 \%$ & $25 \%$ & $21 \%$ & $24 \%$ \\
\hline $\begin{array}{l}\text { Pourcentage d'eau dans la plante (water content in the } \\
\text { plant) }\end{array}$ & $41 \%$ & $51 \%$ & $38 \%$ & $50 \%$ \\
\hline
\end{tabular}

* Différence avec le lot EXT significative au seuil de $5 \%$

** Différence avec le lot EXT significative au seuil de $1 \%$.

*** Différence avec le lot EXT significative au seuil de $1 \%$. 
de grains est relativement moins réduite $(-8 \mathrm{p} .100)$. Cette diminution du rendement provient surtout d'une diminution du nombre de grains par épi (les grains eux-mêmes sont lourds en moyenne) qui serait provoquée par le brusque changement de climat lors de la mise en enceinte.

Dans l'ensemble, les différences entre les plantes cultivées en enceinte et celles restées dehors semblent provenir essentiellement des différences de disponibilité en eau. Dans les expériences ultérieures, les plantes qui resteront à l'extérieur recevront une irrigation identique à celle des plantes en enceinte de façon à éliminer l'effet des différences d'apport d'eau et à savoir si il y a un effet de la température et de l'éclairement.

\section{CONCLUSION}

Nous avons vu que, pour la variété étudiée, la fumure carbonée tout au long de la période explorée (montaisonmaturité) favorise la production de matière sèche de la partie aérienne. Si l'on admet, avec de nombreux auteurs, que la fumure carbonée se traduit d'abord par un accroissement de la fixation photosynthétique du $\mathrm{CO}_{2}$ par la plante, on peut conclure que la photosynthèse est un facteur limitant de la production, qu'elle agisse directement (fourniture d'assimilats) ou qu'elle permette le fonctionnement d'un grand nombre de grains. Le système est donc en grande partie contrôlé par la performance du capteur, lequel agit notamment sur la constitution d'un nombre plus ou moins grand d'unités de stockage.

Une autre conclusion peut être tirée de l'observation du parallélisme entre l'accroissement de la production de grains et celle de la paille. En effet, le progrès apporté par l'amélioration génétique chez l'espèce blé s'est traduit par un accroissement de la production de grains corrélé à une diminution de la production de paille. Cet antagonisme entre la production de grains et celle de paille semble avoir atteint aujourd 'hui ses limites. La répartition des assimilats et en particulier la redistribution de la matière sèche vers les épis pendant la dernière phase de croissance des grains ne peuvent guère être modifiées dans les variétés actuelles, semble-t-il.

Par ailleurs, les expériences entreprises ont montré les limites de la simulation climatique en enceinte pour l'espèce blé, dès lors que l'on travaille sur des plantes adultes. Les plantes témoins placées en enceinte ont en effet une production totale plus élevée que les plantes laissées à l'extérieur. La différence provient, semble-t-il, du niveau d'éclairement plus élevé en enceinte et des difficultés de simuler les «stress " hydriques modérés à effet cumulatif observés à l'extérieur. Les conclusions tirées pour ce qui concerne la fumure carbonée ne s'appliquent, en toute rigueur, qu'à des plantes placées dans des conditions très favorables.

Il n'est donc pas possible actuellement de conclure définitivement : de nouvelles expériences sont entreprises pour étudier simultanément la croissance et l'aptitude photosynthétique des différents organes (feuilles, épis) de blé soumis à des traitements temporaires de fumure carbonée.

\section{REMERCIEMENTS}

L'auteur tient à remercier M. Philippe Auriau pour ses conseils dans le choix du cultivar, les méthodes de culture et la discussion des résultats et $\mathrm{M}$. André KoBILINSKy pour son aide dans l'analyse statistique des résultats. Il remercie également $M$. Jean-Michel BerTolini pour la mise au point du système de régulation de la teneur en $\mathrm{CO}_{2}$ des enceintes $\mathrm{C}_{2}-3 \mathrm{~A}$ et $\mathrm{M}$. Bernard JAussely pour son assistance technique.

Reçu le 28 mai 1980. Accepté le 18 novembre 1980.

\section{RÉFÉRENCES BIBLIOGRAPHIQUES}

Baldy C. M., 1974. Quelques réflexions concernant les caractères du rendement des blés. Ann. Amélior. Plantes, 24 (2), 193-199. Chartier P., 1966. Etude théorique de l'assimilation brute de la feuille. Ann. Physiol. Vég., 8 (3), 167-196.

Evans L. T., 1975. Crop physiology. Some case histories. Cambridge University Press, Cambridge, $374 \mathrm{p}$.

Evans L. T., Dunstone R. L., 1970. Some physiological aspects of evolution in wheat. Austr. J. Biol. Sci., 23, 725-741.

Gifford R. M., 1977. Growth pattern, carbon dioxide exchange and dry weight distribution in wheat growing under differing photosynthetic environments. Austr. J. Plant Physiol., 4, 99-110.

Hardman L. L., Brun W. A., 1971. Effect of atmospheric carbon dioxide enrichment at different developmental stages on growth and yield components of soybeans. Crop Sci., 11 (6), 886-888.
Krenzer E., 1974. Source-sink relationships in wheat as affected by photoperiod and $\mathrm{CO}_{2}$ concentration. Thèse Univ. Microfilms, Ann Arbor (Michigan, USA), $50 \mathrm{p}$.

Rabinowitch E. I., 1951. Photosynthesis and related processes, kinetics of photosynthesis. Interscience Pub. New York (USA), $2088 \mathrm{p}$.

Ryle G. J. A., Powell G. E., 1976. Effect of rate of photosynthesis on pattern of assimilate distribution in graminaceous plant. $J$. Exp. Bot., 27, 189-200.

Tollenaar M., 1977. Sink-source relationship during reproductive development in maize. A review. Maydica, 22, $49-75$.

Wardlaw I. F., 1976. Assimilate partitioning : cause and effect. In : "Transport and transfer processes in plants", Wardlaw I. F., Passioura J. B. Ed., Academic Press, New York (USA), 381-391. 